\title{
A POLÍTICA TARIFÁRIA COMO INSTRUMENTO DE GESTÃO DA DEMANDA POR ÁGUA: ESTIMANDO A ELASTICIDADE PREÇO DA DEMANDA NOS MUNICÍPIOS DO RIO GRANDE DO SUL, 2010-2016
}

\author{
Cristiano Ponzoni Ghinis * \\ Adelar Fochezatto ${ }^{\dagger}$ \\ Christian Velloso Kuhn $\ddagger$
}

\begin{abstract}
Resumo
Problemas de abastecimento de água no Brasil têm sido frequentes nos últimos anos, tornando-se alvo importante para políticas públicas. Entre os seus fatores causadores estão a poluição de rios e mananciais e o consumo descontrolado. Este estudo busca estimar a elasticidade preço da demanda por água nos municípios gaúchos no período 2010-2016. Para isso é usado um modelo de dados em painel com informações do SNIS. Os resultados apontam que a política tarifária pode ser usada como um instrumento eficaz para a redução do consumo de água no Rio Grande do Sul.
\end{abstract}

Palavras-chave: elasticidade preço, demanda por água, dados em painel.

\begin{abstract}
Problems of water supply in Brazil have been frequent in recent years, becoming an important target for public policies. Among its causal factors are the pollution of rivers and springs and uncontrolled consumption. This study seeks to estimate the price elasticity of demand for water in the municipalities of Rio Grande do Sul in the period 2010-2016. For this, a panel data model with SNIS information is used. The results indicate that the tariff policy can be used as an effective instrument to reduce water consumption in Rio Grande do Sul.
\end{abstract}

Keywords: price elasticity, water demand, panel data.

JEL classification: D12, Q21, Q01.

DOI: ht tp : / / dx.doi .org/10.11606/1980-5330/ea 156233

\footnotetext{
* Mestre em Economia do Desenvolvimento. Economista do DMAE/PMPA. E-mail: cristianop.ghinis@dmae.prefpoa.com.br

${ }^{\dagger}$ Doutor em Economia. Professor Titular da PUCRS. Pesquisador do CNPq. E-mail: adelar@pucrs.br

$\ddagger$ Doutor em Economia pela UFRGS. Professor do Instituto Profecom. Economista da CEEE-D.

E-mail: cv_kuhn@yahoo.com.br
} 


\section{Introdução}

Há décadas já é conhecido e difundido o fato de que os recursos hídricos do planeta estão se esgotando gradativamente e que, além da poluição dos rios e dos mananciais, o consumo irresponsável e sem fundamentação sustentável no desenvolvimento econômico é um fator relevante na redução da disponibilidade de água. As preocupações com a escassez desse recurso atualmente alcançam os mais diversos segmentos da esfera social, política e econômica, pois os problemas ambientais têm exigido reflexões sobre a sua utilização em todos os países, sejam desenvolvidos ou em desenvolvimento.

Como agravante, a água, dentre os recursos naturais, o mais essencial à vida, encontra-se distribuída geograficamente de forma extremamente desigual, com abundância em algumas regiões e, em outras, completa escassez. Trata-se de um recurso natural cada vez mais limitado e, como tal, tem reconhecido valor econômico, cuja mensuração leva em conta o preço da conservação, da recuperação e da melhor distribuição desse bem, além dos custos de monitoramento da quantidade, bem como da qualidade da água que está à disposição dos usuários e os recursos que devem ser destinados às demais ações de gerenciamento (Albuquerque \& Maia 2008).

No Brasil, entre os anos de 2014 e 2016, tem-se o exemplo da capital de São Paulo, centro econômico e financeiro do país, que vivenciou uma das maiores crises hídricas da sua história. Tal crise ocorreu em função de fatores climáticos, com longos períodos de seca, que, combinados com a falta de planejamento, afetaram seriamente a população com o desabastecimento de água. Nesse cenário, o Rio Grande do Sul, embora tenha maior disponibilidade de recursos hídricos e de oferta de água tratada comparativamente às regiões Norte e Nordeste do país, também tem tido problemas com a escassez desse recurso (o volume médio de água tratada disponibilizada por economia nas regiões Norte e Nordeste foi de 13,26 metros cúbicos ao mês em 2016, ao passo que, no estado, tal volume se situou em 18,92). Como agravante, o Rio Grande do Sul tem acompanhado a tendência nacional de aumento significativo do consumo médio per capita de água nos últimos anos (SNIS - Sistema Nacional de Informações sobre Saneamento 2016).

De qualquer sorte, o marco regulatório sobre o setor de saneamento básico no país (que contempla, dentre outros eixos de serviços, os de abastecimento de água) foi marcado, já nos anos 1960, pela primeira política nacional de saneamento, o Plano Nacional de Saneamento (Planasa), implantado pelo Banco Nacional da Habitação (BNH) em 1968. O BNH abrigou o Sistema Financeiro de Saneamento (SFS) e foi, até meados dos anos 1980, o órgão responsável pela concessão dos financiamentos e pela própria regulação do setor. A partir desse modelo vigente até então, por meio do Decreto 82.587, que regulamentou a Lei $\mathrm{n}^{\circ} 6.528$, de 11 de maio de 1978, estabeleceu-se diretrizes mais claras para a cobrança pelos serviços de saneamento básico (Disponível em: http://www.planalto.gov.br/ccivil_03/decreto/d82587.htm).

Em 1997, a Lei ${ }^{\circ}$ 9.433, que instituiu a Política Nacional de Recursos Hídricos (PNRH), determinou que a cobrança pelo uso de recursos hídricos tem por objetivos: reconhecer a água como um bem econômico e dar ao usuário uma indicação de seu real valor, incentivar a racionalização do uso da água e obter recursos financeiros para o financiamento dos programas e intervenções previstos nos planos de recursos hídricos (Art. 19). Portanto, a cobrança pela utilização da água passou, já a partir da referida lei, a ter como um dos 
objetivos principais no Brasil o estímulo ao consumo responsável, ou, alternativamente, o incentivo à redução do desperdício no consumo. Desse modo, a referida lei traz consigo um significativo avanço nas diretrizes para a condução da política tarifária do setor, qual seja, a utilização da cobrança da água como instrumento de gestão da demanda.

Nessa perspectiva, em 05 de janeiro de 2007, foi criada no Brasil a Lei de Saneamento Básico Federal (LSB) $n^{\circ} 11.445$, que estabeleceu as diretrizes nacionais para o saneamento básico, além do Decreto $\mathrm{n}^{\circ} 7.217$, de 21 de junho de 2010, que a regulamenta. No artigo 29 dessa lei, que descreve sobre os aspectos econômicos e sociais na prestação dos serviços do setor, no $\S 1^{\circ}$ fica estabelecido que a instituição das tarifas, preços públicos e taxas para os serviços de saneamento básico deverá observar, dentre diversas outras diretrizes, a inibição do consumo supérfluo e do desperdício de recursos.

Contudo, a eficácia da utilização da política tarifária como instrumento de gerenciamento da demanda por água pode depender de múltiplos fatores que influenciam a relação entre os preços cobrados e o volume de água consumido, tais como: i) se (e quanto) o nível de consumo está acima daquele que poderia ser considerado como o mínimo necessário para a sobrevivência, e essa é uma medida subjetiva, pois varia para cada indivíduo e em função da própria heterogeneidade socioeconômica e ambiental no Brasil; ii) qual a disponibilidade de água em uma determinada região, dada a sua distribuição desigual no território nacional; iii) qual o peso da agropecuária e alguns segmentos da indústria (por exemplo, alimentos e bebidas) na economia local, pois são setores que utilizam amplamente a água como insumo no processo de produção, podendo apresentar maior sensibilidade aos preços; dentre diversos outros aspectos (Worthington \& Hoffman 2008).

Em outras palavras, a análise da eficácia dessa política como instrumento de gestão do consumo de água depende, principalmente, da elasticidade-preço da demanda por esse bem, isto é, de qual a variação ou o quão sensível é seu o consumo dada uma variação nos seus preços. Como se trata de um bem essencial à vida, espera-se que a demanda por água seja relativamente inelástica ao nível de preços. Porém, tal relação pode não ser perfeitamente inelástica (elasticidade-preço igual à zero). A análise de sensibilidade do nível de consumo de água em relação à sua cobrança é fundamental na orientação de políticas públicas que visem incentivar a racionalização do uso desse bem, principalmente em um cenário de escassez de recursos hídricos. E alguns estudos apontam que, em termos qualitativos, o estado já sofre com a escassez de disponibilidade de água (Célia 2007).

Ademais, como instrumento de gerenciamento interno da política de preços das prestadoras, a análise da significância e da magnitude dessa relação é central, inclusive, para a tomada de decisões quanto à condução da própria política tarifária, dado que, conforme o Decreto $n^{\circ} 7.217$, Artigo 27, Inciso IV, os preços cobrados devem considerar a modicidade tarifária, mas, ao mesmo tempo, assegurar o equilíbrio econômico-financeiro das empresas, de modo a garantir a universalização do acesso da população aos serviços de saneamento básico.

Nesse contexto, o presente trabalho busca investigar a relação entre a política tarifária do setor nos municípios gaúchos e o comportamento da demanda por água, estimando-se o quão sensível tem se mostrado o seu consumo em função de variações no nível de preços. Isto é, propõe-se mensurar a elasticidade-preço da demanda do setor no Rio Grande do Sul. Além disso, 
dada a heterogeneidade ambiental e socioeconômica das regiões do estado, procura-se estimar também a elasticidade da demanda por água especificamente na capital gaúcha, para verificar se existe diferença significativa em relação aos demais municípios do Rio Grande do Sul. Para tanto, utiliza-se principalmente a base de dados do Sistema Nacional de Informações sobre Saneamento (SNIS) para todos os municípios gaúchos no período 2010-2016. E, com estes, busca-se a especificação e a mensuração de parâmetros a partir da análise de dados em painel, considerando os cortes seccional e temporal supracitados.

Este trabalho foi organizado em 5 seções. Além da presente introdução, na segunda seção, busca-se analisar algumas características municipais do consumo de água, enfatizando-se a capital e o Rio Grande do Sul no contexto nacional. Na seção seguinte, apresentam-se a metodologia e a especificação das variáveis do modelo de dados em painel a ser estimado. Na quarta, demonstramse os principais resultados dos parâmetros estimados no Rio Grande do Sul, e se testa se há diferença significativa na capital gaúcha. Por fim, na quinta seção, apresentam-se as considerações finais do trabalho.

\section{Caracterização da Demanda por Água no Território Gaúcho}

O Brasil como um todo sempre se caracterizou pela abundância de recursos naturais, dentre os quais a água, comparativamente aos países desenvolvidos. Contudo, ao longo dos anos, a expansão das atividades econômicas sem base no crescimento sustentável, com tecnologias de produção que não previam a otimização do consumo de água e a minimização de emissão de potenciais poluidores; o crescimento populacional nas capitais brasileiras sem contrapartida da redução do consumo per capita, com base no consumo responsável (ao contrário, conforme dados do SNIS de 2001 a 2016, houve um sensível aumento do volume consumido por habitante em várias regiões do país ao longo dos anos); a expansão da construção civil, acelerando o número de domicílios ocupados e o próprio processo de urbanização, principalmente em contextos de redução dos juros e expansão do crédito; dentre diversos outros fatores, têm contribuído para a redução da disponibilidade e qualidade da água no país. Isso inclui as grandes capitais.

No contexto nacional, em contraste aos municípios da região Nordeste, o Rio Grande do Sul sempre se caracterizou pela disponibilidade de recursos hídricos. A Figura 1 mostra a oferta, medida pelo volume de água disponibilizado por unidade consumidora, e a demanda, mensurada pelo volume de água consumido por economia ( $\mathrm{m}^{3} / \mathrm{mês} /$ economia), no país, no estado e em Porto Alegre. Observa-se que, tanto o Rio Grande do Sul, quanto a capital, em maior medida, de fato, caracterizam-se pelo volume superior de água tratada disponibilizada em relação à demanda por economia. Mais precisamente, no Brasil, no estado e na capital, o consumo representou, respectivamente, 58,3\%, $56,4 \%$ e $61,1 \%$ da disponibilidade de água tratada por economia em 2016 , segundo dados do SNIS. Isso não significa que não haja o comprometimento cada vez maior da disponibilidade de recursos hídricos, em função da própria escassez em termos da qualidade dos mesmos. Mas, em alguns estados da região Norte, por exemplo, em Roraima e Tocantins, tal percentual atingiu 81,9 e $83,3 \%$, respectivamente, no mesmo ano.

A Figura 2 mostra a evolução da oferta e da demanda de água por econo- 
Figura 1: Disponibilidade e consumo de água por economia no Brasil, Rio Grande do Sul e Porto Alegre (m3/mês/economia) - 2016

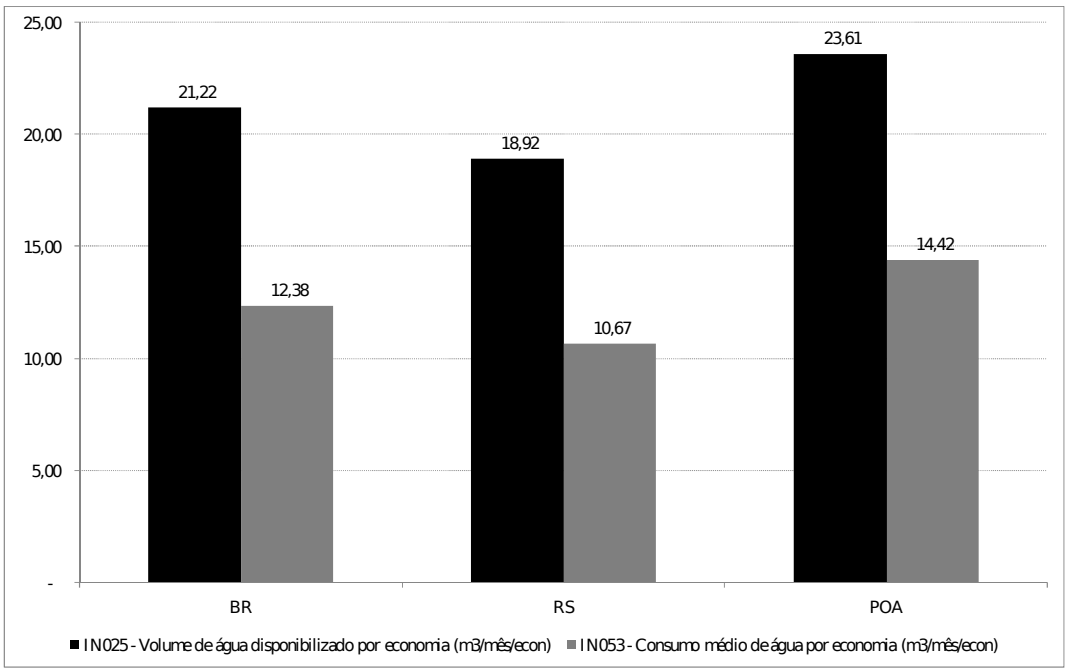

Fonte: Sistema Nacional de Informações sobre Saneamento (SNIS)

mia e por habitante no Rio Grande do Sul nos anos 2001-2016. Observa-se que o volume de água disponibilizado por economia ( $\mathrm{m} 3 / \mathrm{mês} /$ economia) tem registrado sucessivas quedas ao longo dos anos 2001-2016, mais precisamente, de $-29,5 \%$ em todo o período. Tais decréscimos têm sido acompanhados, porém em menor proporção, pelo comportamento da demanda, com uma diminuição de $-24,6 \%$ na média do volume consumido por economia nos mesmos anos. Em contraste, quando se analisa a tendência da demanda pelo consumo médio per capita de água, nota-se que o consumo caiu até 2005 , mas voltou a crescer significativamente a partir de então e se estabilizou em um patamar muito próximo ao ano base de 2001 no último ano em estudo, 2016.

Comparativamente ao Brasil, o comportamento do consumo médio per capita do Rio Grande do Sul seguiu praticamente a mesma tendência nacional, principalmente nos últimos anos, quando a demanda, medida por esse indicador, teve um comportamento crescente (Figura 3). Chama a atenção, no entanto, que, considerando a capital gaúcha, o consumo médio per capita de Porto Alegre sempre esteve substancialmente acima das médias apresentadas no estado e no país. Em 2001, esta já se estabelecia em 236,5 litros/habitante/dia. E, em 2016, apesar da suave queda em relação ao ano inicial, ainda permaneceu em 220,3 litros/habitante/dia, ficando superior às médias do país e do estado em $43,5 \%$ e $48,5 \%$, respectivamente. Segundo informações do SNIS, em 2016 Porto Alegre registrou a segunda maior média de consumo per capita dentre as capitais brasileiras, ficando abaixo apenas da cidade do Rio de Janeiro, cujo consumo foi de $328,9^{1}$ litros/habitante/dia (SNIS - Sistema Nacional de Informações sobre Saneamento 2016).

Como se observa na Figura 4 (a), nos demais municípios gaúchos, a maior concentração de cidades ocorreu em 2016, nas faixas de consumo ainda abaixo da média da capital, quais sejam: 232 municípios, ou 49,9\% do total das

\footnotetext{
${ }^{1}$ Um dos fatores que podem contribuir na explicação do elevado consumo na capital carioca, comparativamente a Porto Alegre, é a maior proporção de economias não residenciais no total de unidades de consumo.
} 
Figura 2: Indicadores de consumo e disponibilidade da água no Rio Grande do Sul - 2001-2016

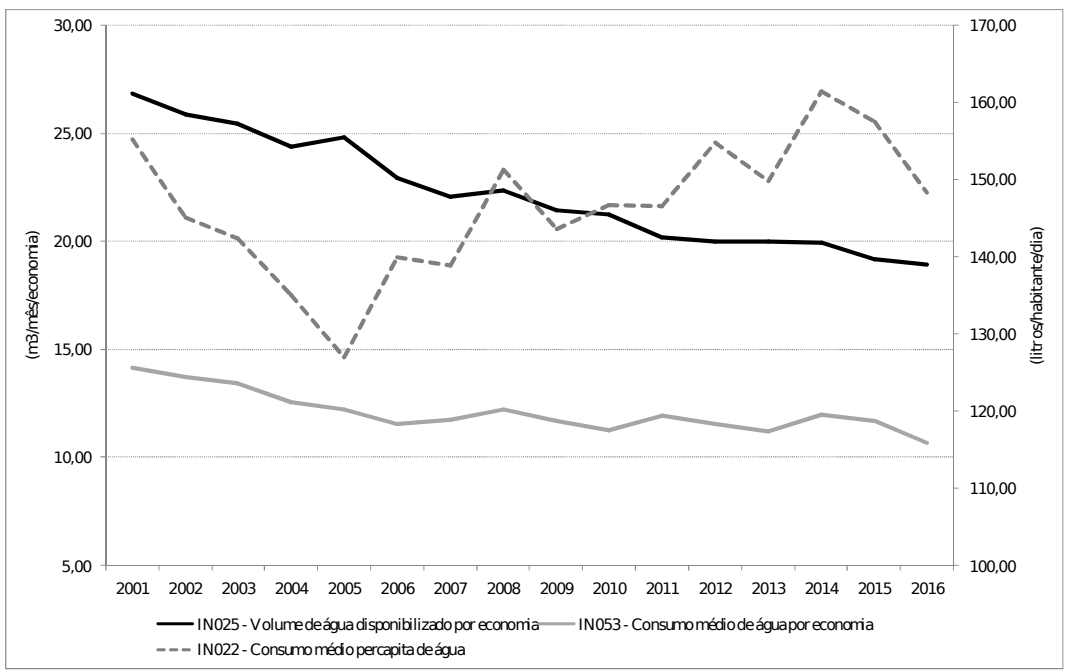

Fonte: Sistema Nacional de Informações sobre Saneamento (SNIS)

Figura 3: Consumo médio per capita no Brasil, Rio Grande do Sul e Porto Alegre (litros/habitante/dia) - 2001-2016

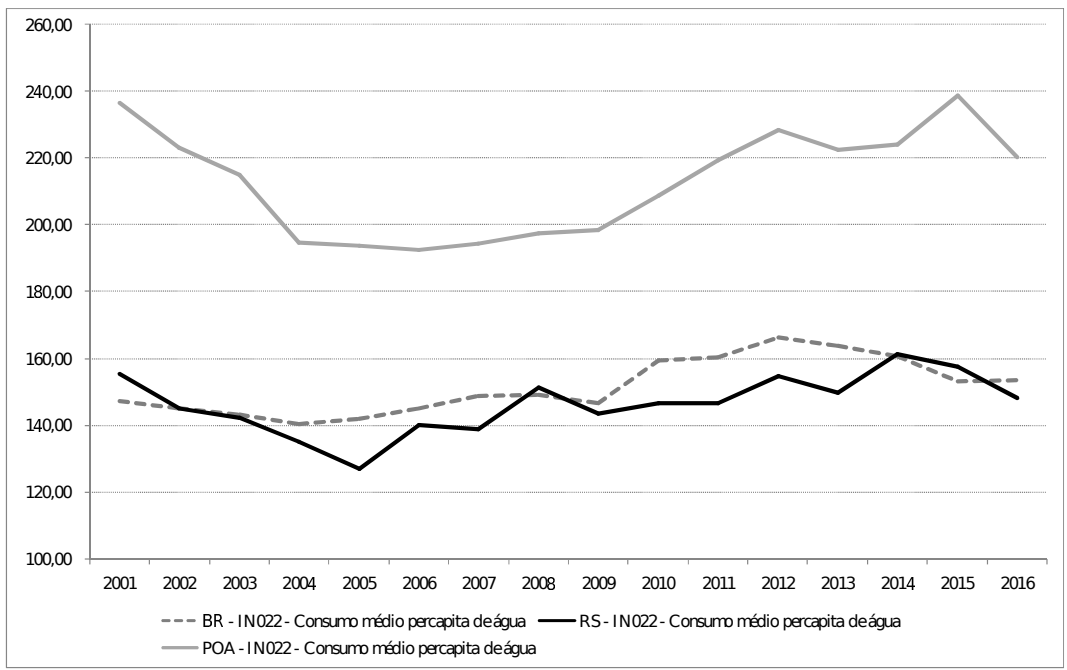

Fonte: Sistema Nacional de Informações sobre Saneamento (SNIS) 
cidades gaúchas, situaram-se na faixa de consumo médio per capita (entre 118 e 177,1 litros/habitante/dia) e 174 municípios (totalizando 87,3\% das cidades do estado) registraram consumo entre 59 e 118 litros/habitante/dia ${ }^{2}$. $\mathrm{Na}$ faixa de consumo em que se inclui a capital gaúcha, entre 177 e 236 litros/habitante/dia, houve concentração de 33 municípios do estado, somando $94,4 \%$ das cidades. De outra parte, menores concentrações puderam ser observadas nas faixas de 236 a 590 litros/habitante/dia, totalizando 5,6\% restantes do total de municípios gaúchos ${ }^{3}$.

Sob o ponto de vista do consumo em relação à disponibilidade de água tratada por economia (isto é, da proporção da oferta destinada ao consumo das economias), embora com menor concentração, destacaram-se as faixas de consumo médio per capita entre $50 \%$ e $60 \%, 61 \%$ e $70 \%$ e $71 \%$ e $80 \%$, as quais, conjuntamente, representaram mais da metade dos municípios gaúchos (especificamente, 56,1\% do total das cidades). Além destes, 124 cidades apresentaram faixas preocupantes de $80 \%$ e $90 \%$, e $91 \%$ e $100 \%$, o que representa $24,6 \%$ dos municípios do estado, acumulando $80,7 \%$ das cidades (Figura 4 (b)). Ou seja, embora o Rio Grande do Sul não se caracterize, historicamente, pela escassez de recursos hídricos, parcelas significativas de municípios gaúchos têm registrado elevados níveis de consumo proporcionalmente à disponibilidade de água tratada.

O Apêndice 1 apresenta um mapa quantílico, que exibe distribuições de categorias de consumo médio per capita com igual número de observações de municípios, em 5 faixas aproximadas a cada 100 municípios gaúchos. Observase que os maiores consumos ocorreram, em 2016, nas cidades de Erechim, Westfalia, Caxias do Sul, Bento Gonçalves, Canela, Triunfo, Gramado, dentre outros, além da capital gaúcha. Estes, majoritariamente, conforme dados do SNIS, apresentaram maiores proporções do número de unidades consumidoras não residenciais no total de economias, à exceção de Porto Alegre e alguns outros municípios ${ }^{4}$. Em oposição, naquelas cidades cujo consumo foi predominantemente residencial, observaram-se as menores médias per capita do indicador, a saber: Charrua, Pontão, Engenho Velho, Alto Feliz, Novo Tiradentes, Barra Funda, Capão Bonito do Sul, dentre outras. De outra parte, em síntese, como se observa no Apêndice 2, que mostra o consumo em relação à disponibilidade de água tratada por economia, a distribuição do indicador no território gaúcho é muito similar ao caso anterior. Isto é, em grande medida, as cidades gaúchas que apresentaram maiores médias de consumo estão, de fato, comprometendo parcelas significativas da oferta de água tratada.

\section{Metodologia}

A elasticidade preço da demanda (Ep) é um conceito bem difundido na teoria econômica. Ela mede a variação proporcional na quantidade demandada de um determinado bem ou serviço em função de uma variação proporcional

\footnotetext{
${ }^{2}$ Conforme informações da Organização Mundial da Saúde (OMS), o consumo mínimo diário, considerando todas as necessidades humanas no uso da água, situa-se em torno de 110 litros/habitante/dia (Fonte: https://www.who.int/eportuguese/countries/bra/pt/).

${ }^{3}$ Nesses casos, cabe salientar que se observa pesos maiores da agropecuária e de alguns segmentos da indústria na estrutura produtiva econômica local.

${ }^{4}$ Em 2016, o município com maior consumo médio per capita foi Vale Real, com 590,23 litros/habitante/dia. Porém, é importante frisar que o baixo índice de micromedição, segundo os próprios dados do SNIS, gera incertezas nesse caso.
} 
Figura 4: Figura 1 - Distribuição de frequências do consumo per capita e do consumo em relação a disponibilidade de água nos municípios gaúchos - 2016.

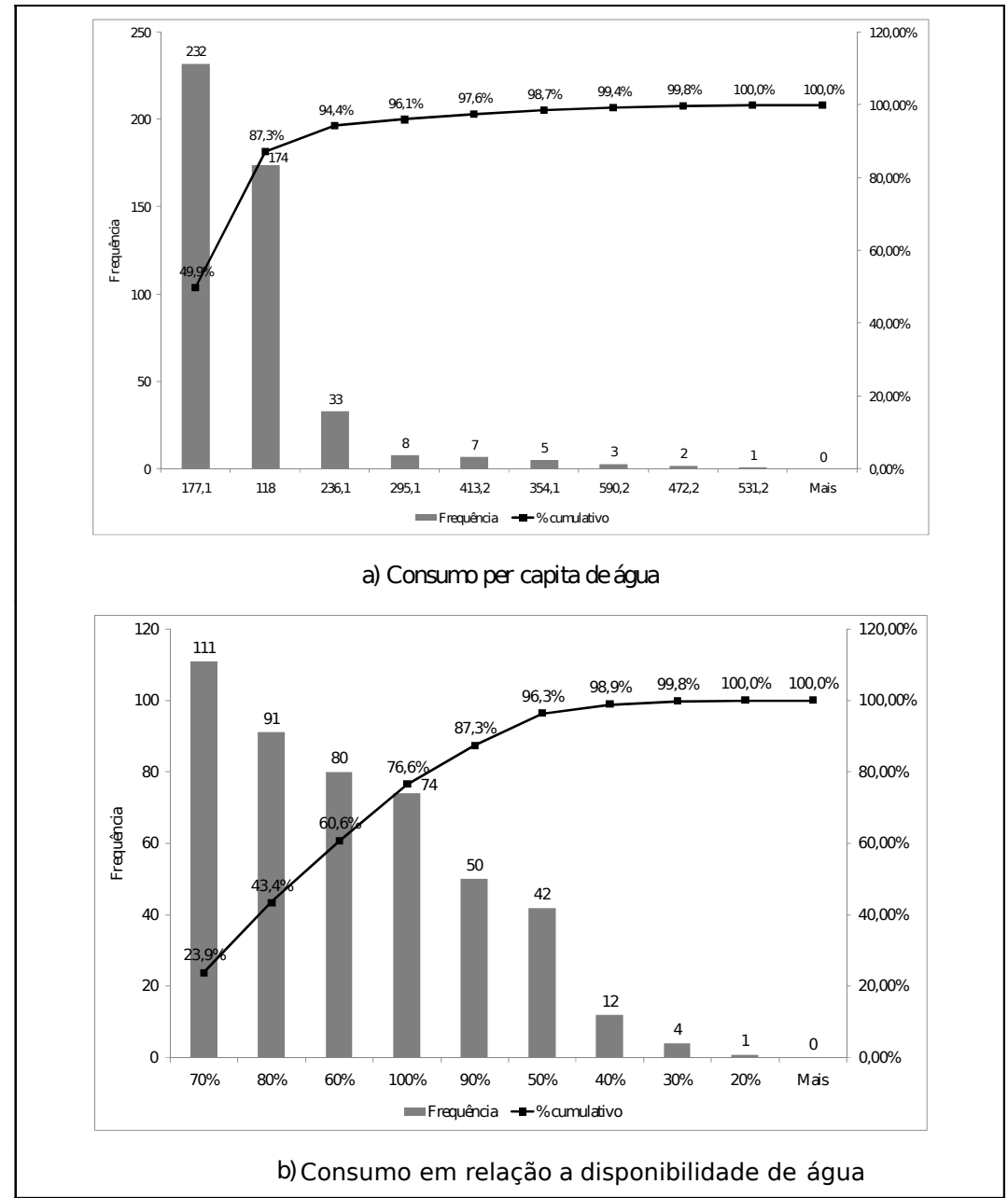

Fonte: Sistema Nacional de Informações sobre Saneamento (SNIS) 
no seu preço. Em outras palavras, ela mede o quão sensível é a demanda em função de variações no nível de preços. Ela tende a ser negativa, isto é, aumentos nos preços proporcionam a redução na quantidade demandada. E pode ser representada como:

$$
E p=\frac{\Delta Q / Q}{\Delta P / P}
$$

em que: Ep é a elasticidade-preço da demanda; Q é a quantidade demandada e $\mathrm{P}$, o preço do bem. Quando Ep $<-1$ (ou, em módulo, $|\mathrm{EP}|>1$ ), variações no preço tendem a diminuir a quantidade demanda em maior proporção, ou seja, a demanda é elástica aos preços. Se Ep $>-1$ (ou, em módulo, $|\mathrm{EP}|<1$ ), aumentos no preço tendem a reduzir a demanda em menor magnitude, sendo a demanda inelástica. Caso $\mathrm{Ep}=0$, isso significa que variações no preço não geram alterações na quantidade demandada, ou a demanda é perfeitamente inelástica. Já, se $\mathrm{Ep}=\alpha$, ou seja, se a elasticidade tende ao infinito, por exemplo, no caso de bens substitutos perfeitos, a demanda é perfeitamente elástica (Varian 1990).

Como a água é um bem essencial a vida, pode-se esperar que a sua demanda seja inelástica ao nível de tarifas cobradas. Contudo, tal relação pode não ser perfeitamente inelástica. Dada a sua importância vital e o contexto de cada vez maior preocupação mundial quanto à disponibilidade de recursos hídricos, diversos trabalhos têm buscado estudar a relação empírica entre os preços e a demanda por esse bem. Worthington \& Hoffman (2008) fizeram uma compilação desses estudos empíricos aplicados nos últimos 25 anos por diversos autores nos Estados Unidos. Os autores concluíram que, em grande parte deles, as variáveis utilizadas nos modelos foram relacionadas aos preços e à estrutura tarifária, aos níveis de renda, às condições climáticas e ambientais que determinaram componentes sazonais no consumo, ao crescimento populacional e do número de domicílios, aos níveis de educação e a outras variáveis não monetárias que impactam nos padrões culturais de demanda. De um modo geral, os estudos concluíram que a elasticidade preço da demanda para o país como um todo tem sido estimada com coeficientes negativos em torno de 0,5 , ou seja, aumentos das tarifas de $1 \%$ podem ter induzido quedas no consumo em torno de $0,5 \%$.

Em termos regionais, já no início da década de 70, o trabalho de Wong (1972) buscou analisar a relação entre política tarifária e o consumo de água nos municípios do nordeste de Illinois. Como principal conclusão, o estudo encontrou elasticidade preço da demanda pelo bem que variaram de $-0,02$ a $-0,82$, com resultados estatisticamente significativos na maioria dos municípios. Young (1973), em semelhante abordagem, testou a significância na relação entre demanda e tarifas de água no caso de Tucson, cidade do sudoeste do Arizona. O autor chegou à estimativa de elasticidade preço do consumo de água situada em - 0,42. Foster \& Beattie (1979) estudaram essa relação para o consumo de municípios predominantemente urbanos dos Estados Unidos, estimando resultados que alcançaram entre - 0,30 e - 0,69, para coeficientes significativos dessa elasticidade. Schneider \& Whitlach (1991), neste enfoque, mas investigando especificamente o consumo residencial de água, estimaram esse parâmetro na faixa de - 0,26 para o município de Columbus, no estado de Ohio. Tais trabalhos mostram a importância de se estudar essa relação, pois, apesar das evidências de que a demanda é inelástica ao preço, na grande 
maioria dos casos, ela se mostrou diferente de zero e significativa, revelando ser este um instrumento válido para práticas de políticas públicas que visam estimular a responsabilidade no consumo.

Ademais, no Brasil, observa-se a existência de poucos trabalhos com tal abordagem. Por exemplo, Rosa et al. (2006) estimam tal parâmetro para o caso do estado do Ceará. Os autores, utilizando dados em corte seccional para os municípios do estado, estimam um coeficiente negativo de -0,355, considerando o consumo residencial urbano de água. Já Amaral (2000), com base em um modelo de séries temporais, conclui que o consumo de água em Piracicaba pode ser explicado principalmente pelo consumo passado, observando componentes de persistência e sazonalidade da variável ao longo tempo. Outro ponto a ser considerado, portanto, é que o presente estudo utiliza um modelo de dados em painel, ainda pouco explorado para esse tema no país.

Neste trabalho, propõe-se a aplicação de um modelo com o uso de dados em painel, no qual todos os municípios gaúchos com informações declaradas no SNIS foram observados ao longo do período 2010-2016. Tal corte temporal se justifica em função da disponibilidade de dados municipais nas fontes de informações utilizadas, as quais são a seguir demonstradas.

Os modelos de dados em painel combinam dados de séries temporais e em corte seccional, tendo como vantagem, em geral, um grande número de graus de liberdade na estimação dos parâmetros (Coelli et al. 1998).

Entre os modelos de dados em painel mais utilizados estão: o Modelo de Regressões Aparentemente Não Relacionadas (Seemingly Unrelated Regressions - SUR), o Modelo de Efeitos Fixos e o Modelo de Efeitos Aleatórios. O Modelo SUR supõe que o intercepto e os parâmetros de resposta a serem estimados diferem entre os indivíduos, mas são constantes no tempo. E, por isso, possui uma limitação. Quando o número de indivíduos é muito grande o número de parâmetros a ser estimado também será grande, restringindo os graus de liberdade na estimação dos parâmetros e podendo levar a estimativas pouco fidedignas do modelo em questão.

O Modelo de Efeitos Fixos busca controlar os efeitos das variáveis omitidas que variam entre indivíduos e permanecem constantes ao longo do tempo. Assim, supõe que o intercepto varia de um indivíduo para outro, porém é constante ao longo do tempo, ao passo que os parâmetros de resposta a serem estimados são constantes para todos os indivíduos e em todos os períodos de tempo (Hill et al. 1999). Ele é a melhor opção para modelar os dados em painel quando o intercepto é correlacionado com as variáveis explicativas, seja qual for o período de tempo (Wooldridge 2002).

O Modelo de Efeitos Aleatórios (Variáveis) possui as mesmas suposições do Modelo de Efeitos Fixos, ou seja, o intercepto varia de um indivíduo para outro, mas não ao longo do tempo, sendo os parâmetros de resposta constantes para todos os indivíduos e em todos os períodos de tempo. No entanto, a diferença entre os dois modelos refere-se ao tratamento do intercepto. Enquanto o Modelo de Efeitos Fixos trata o intercepto como parâmetros fixos, o de efeitos aleatórios trata o intercepto como variáveis aleatórias. Isto é, considera que os indivíduos para os quais se dispõem de dados são amostras aleatórias de uma população maior de indivíduos (Hill et al. 1999).

Conforme Wooldridge (2002) e Asteriou \& G (2007), o principal determinante para a escolha do modelo a ser utilizado é o efeito não observado, ou seja, a diferenciação do intercepto entre os indivíduos. Quando ele não é correlacionado com as variáveis explicativas, o Modelo de Efeitos Aleatórios é o 
mais indicado. Em contraste, quando ele for correlacionado com algumas variáveis explicativas, então o Modelo de Efeitos Fixos deve ser utilizado. Para testar tal correlação, segundo os autores, deve-se utilizar o Teste de Hausman. Neste estudo, os resultados para esse teste apontaram para a escolha do Modelo de Efeitos Fixos. Portanto, a equação a ser estimada, com base nos estudos empíricos anteriormente abordados, foi definida como:

$$
\begin{aligned}
\ln \left(D_{A i t}\right)=C_{i}+ & \beta_{1} \ln \left(P_{i t}\right)+\beta_{2} \ln \left(D O M_{i t}\right)+\beta_{3} \ln \left(P O P_{i t}\right)+\beta_{4} \ln \left(T_{i t}\right) \\
+ & \beta_{5} \ln \left(I N D_{i t}\right)+\beta_{6} \ln \left(A G R O_{i t}\right)+\beta_{7} \ln \left(E S C 1_{i t}\right) \\
& +\beta_{8} \ln \left(E S C 2_{i t}\right)+\beta_{9} \ln \left(E S C 3_{i t}\right)+\beta_{10} \ln \left(R E M_{i t}\right) \\
& +\beta_{11} \ln \left(D P O A * P_{i t}\right)+u_{1 i t}
\end{aligned}
$$

em que: $D_{A}=$ volume de água consumido, em metros cúbicos; $C=$ constante estimada; $P=$ tarifa média de água cobrada pelos serviços, em reais por metro cúbico faturado, a preços constantes de 2016, usando como deflator o Índice de Preços ao Consumidor Amplo (IPCA) ${ }^{5}$; DOM = número de domicílios; $P O P=$ taxa de crescimento populacional; $T$ = temperatura média anual; $I N D=$ taxas de crescimento reais da produção do setor industrial ${ }^{6} ; A G R O=$ taxas de crescimento reais da produção do setor agropecuário ${ }^{7} ; E S C_{1}=$ proporção da população economicamente ativa, medida a partir do emprego formal, classificada como analfabeta; $E S C_{2}$ = proporção da PEA, medida pelo emprego formal, com nível fundamental completo; $E S C_{3}=$ proporção da PEA com, no mínimo, ensino médio incompleto (diversos estudos têm comprovado que o nível educacional da população adulta é estatisticamente significante na determinação do comportamento do consumo); REM = PIB municipal per capita, a preços constantes de 2016, usando como deflator o Índice Geral de Preços de Mercado (IGP-M); DPOA = variável dummy que assume o valor 1 quando se trata do município de Porto Alegre; $u_{1}=$ termo de erro aleatório que captura a influência de outras variáveis não especificadas no modelo; $l n=$ logaritmo natural; $i$ e $t=$ notações que indicam que as observações se referem ao município " $i$ " (corte seccional) e período " $t$ " (corte temporal anual). Cabe enfatizar, novamente, que todas as informações constantes nesse modelo foram utilizadas por município e por ano, no período 2010-2016, com dados disponíveis nas bases cujas fontes são mostradas na Tabela 1.

Teoricamente, e como alguns estudos têm comprovado, espera-se que o aumento do preço possa gerar uma diminuição no consumo de água. Em relação às demais variáveis, espera-se que o crescimento populacional e do número de domicílios tenda a gerar um aumento do consumo de água, assim como elevações na temperatura média. Como uma parcela significativa na demanda por água é dada em função da agricultura e alguns segmentos da indústria (principalmente de bebidas), o consumo de água também pode estar diretamente relacionado com essas variáveis. Ademais, dada a conscientização educacional, quanto maior o nível de educação dos indivíduos, espera-se que menor

\footnotetext{
${ }^{5} \mathrm{O}$ IPCA é o indexador de tarifas de água mais utilizado nas 30 maiores prestadoras desse serviço no Brasil.

${ }^{6} \mathrm{~A}$ escolha dessa variável reside no fato de parcela significativa do consumo de água ser da indústria.

${ }^{7}$ Em diversos municípios predominantemente rurais no Brasil, o consumo de água é determinado, principalmente, pelo ritmo de expansão das atividades agropecuárias.
} 
Tabela 1: Fontes dos dados utilizados no modelo por variável

\begin{tabular}{|c|c|c|c|}
\hline Dados & Fonte & Disponível em: & Acesso em: \\
\hline$D_{A}$ & $\begin{array}{l}\text { Sistema Nacional de Infor- } \\
\text { mações sobre Saneamento }\end{array}$ & $\begin{array}{l}\text { http://www.snis.gov.br/aplicacao-web- } \\
\text { serie-historica }\end{array}$ & $18 / 12 / 2018$ \\
\hline$P$ & $\begin{array}{l}\text { Sistema Nacional de Infor- } \\
\text { mações sobre Saneamento }\end{array}$ & $\begin{array}{l}\text { http://www.snis.gov.br/aplicacao-web- } \\
\text { serie-historica }\end{array}$ & $18 / 12 / 2018$ \\
\hline $\operatorname{DOM}\left({ }^{*}\right)$ & $\begin{array}{l}\text { Instituto Brasileiro de Geo- } \\
\text { grafia e Estatística - IBGE / } \\
\text { Pesquisa Nacional por Amos- } \\
\text { tra de Domicílios - PNAD }\end{array}$ & $\begin{array}{l}\text { https://www.ibge.gov.br/estatisticas- } \\
\text { novoportal/downloads-estatisticas.html }\end{array}$ & $18 / 12 / 2018$ \\
\hline POP & $\begin{array}{l}\text { Instituto Brasileiro de Geo- } \\
\text { grafia e Estatística - IBGE / } \\
\text { Censo Demográfico } 2010 \text { e } \\
\text { Estimativas de População }\end{array}$ & $\begin{array}{l}\text { https://www.ibge.gov.br/estatisticas- } \\
\text { novoportal/downloads-estatisticas.html }\end{array}$ & $18 / 12 / 2018$ \\
\hline$T$ & $\begin{array}{l}\text { Ministério da Agricultura, } \\
\text { Pecuária e Abastecimento / } \\
\text { Instituto Nacional de Meteo- } \\
\text { rologia - INMET / Banco de } \\
\text { Dados Meteorológicos para } \\
\text { Ensino e Pesquisa - BDMEP }\end{array}$ & $\begin{array}{l}\text { http://www.inmet.gov.br/portal/index.php?r= } \\
\text { bdmep/bdmep }\end{array}$ & $23 / 12 / 2018$ \\
\hline
\end{tabular}


Tabela 1: Fontes dos dados utilizados no modelo por variável (continuação)

\begin{tabular}{|c|c|c|c|}
\hline Dados & Fonte & Disponível em: & Acesso em: \\
\hline$I N D$ & $\begin{array}{l}\text { Instituto Brasileiro de Geo- } \\
\text { grafia e Estatística - IBGE / } \\
\text { PIB Municípios }\end{array}$ & $\begin{array}{l}\text { http://downloads.ibge.gov.br/downloads_ } \\
\text { estatisticas.htmPIB }\end{array}$ & $23 / 12 / 2018$ \\
\hline$A G R O$ & $\begin{array}{l}\text { Instituto Brasileiro de Geo- } \\
\text { grafia e Estatística - IBGE / } \\
\text { PIB Municípios }\end{array}$ & $\begin{array}{l}\text { http://downloads.ibge.gov.br/downloads_ } \\
\text { estatisticas.htmPIB }\end{array}$ & $23 / 12 / 2018$ \\
\hline $\begin{array}{l}E S C 1 \\
E S C 2 \mathrm{e} \\
E S C 3\end{array}$ & $\begin{array}{l}\text { Instituto Brasileiro de Geo- } \\
\text { grafia e Estatística - IBGE } \\
\text { / Censo Demográfico } 2010 \\
\text { e Estimativas de População; } \\
\text { e Ministério do Trabalho e } \\
\text { Emprego - MTE/ Relação } \\
\text { Anual de Informações Soci- } \\
\text { ais - RAIS }\end{array}$ & $\begin{array}{l}\text { https://www.ibge.gov.br/estatisticas- } \\
\text { novoportal/downloads-estatisticas.html } \\
\text { http://www.rais.gov.br/sitio/index.jsf }\end{array}$ & $23 / 12 / 2018$ \\
\hline$R E M$ & $\begin{array}{l}\text { Instituto Brasileiro de Geo- } \\
\text { grafia e Estatística - IBGE / } \\
\text { PIB Municípios }\end{array}$ & $\begin{array}{l}\text { http://downloads.ibge.gov.br/downloads_ } \\
\text { estatisticas.htmPIB }\end{array}$ & $23 / 12 / 2018$ \\
\hline Deflator & $\begin{array}{l}\text { Instituto Brasileiro de Geo- } \\
\text { grafia e Estatística - IBGE/ } \\
\text { Î́ndice de Preços ao Consu- } \\
\text { midor Amplo (IPCA). }\end{array}$ & $\begin{array}{l}\text { https://www.ibge.gov.br/estatisticas- } \\
\text { novoportal/downloads-estatisticas.html }\end{array}$ & $18 / 12 / 2018$ \\
\hline
\end{tabular}


tenda a ser o consumo de água, sendo que relação similar pode ocorrer com respeito aos níveis de renda (Worthington \& Hoffman 2008).

Assim, em termos do modelo especificado, como resultado principal, o coeficiente $\beta_{1}$ estimado dará o sentido (relação direta, caso positivo, ou inversa, quando negativo, sendo que, conforme o esperado, o aumento de preço de um bem normal tende a reduzir o seu consumo) e a magnitude dos efeitos de variações no preço sobre alterações percentuais da demanda dos serviços. Se $\beta_{1}=0$ (ou próximo de zero), isso significa que mudanças nos preços não afetam significativamente o nível de consumo dos serviços. Quando $\beta_{1}=1$, ou próximo da unidade, isso quer dizer que um aumento na tarifa gera, em média, uma redução de consumo praticamente na mesma proporção. Se $\beta_{1}<1$, pode-se inferir que, em média, mudanças na política tarifária impactam a demanda em menor proporção (ou seja, o consumo de água tende a ser inelástico aos preços). E o inverso ocorre quando $\beta_{1}>1$, pois variações tarifárias podem causar mudanças no consumo em maior magnitude, sendo a demanda elástica às tarifas cobradas. Ademais, dada a heterogeneidade municipal das características ambientais, socioeconômicas e, por conseguinte, do próprio consumo, com o parâmetro $\beta_{11}$, estima-se se há diferença significativa nessa relação para a capital gaúcha. E este tem a mesma interpretação de $\beta_{1}$.

Um dos problemas geralmente encontrados na estimação de modelos de demanda é a presença de viés de simultaneidade. Nos modelos de equações simultâneas, estão envolvidas mais de uma variável dependente, ou endógena, tornando-se necessárias tantas equações quantas forem o número de variáveis endógenas. No caso das curvas de demanda, os preços podem afetar tanto a quantidade demandada quanto a ofertada de um determinado bem, no caso, a água. Sob a hipótese de simultaneidade, a variável explanatória endógena se torna estocástica e está geralmente correlacionada com o termo de erro. Nesse caso, o método clássico de mínimos quadrados ordinários (MQO) tende a gerar estimadores não consistentes, que não convergem para os seus verdadeiros valores populacionais. Por outro lado, caso não haja simultaneidade, tais estimadores serão consistentes e eficientes (Gujarati 2006).

O primeiro passo é então testar a presença de viés de simultaneidade. Caso esse viés esteja presente, a literatura aponta algumas alternativas para resolver o problema. Por exemplo, Pizaia \& Alves (2008) utilizaram mais de um método de estimação dos modelos de demanda. O primeiro foi o próprio método de mínimos quadrados ordinários (MQO); o segundo o método MCFADDEN; e o terceiro e quarto foram os métodos de estimação de equações simultâneas - método de mínimos quadrados em dois estágios (MQ2E) e método da variável instrumental (MVI). No MQ2E, exige-se três passos. O primeiro passo é a estimação do preço como função de todas as variáveis exógenas do sistema. O segundo é a obtenção, então, do valor estimado do preço marginal. E o terceiro passo é a estimativa da equação de demanda, novamente, com o preço marginal estimado. O MVI utiliza uma matriz de Variáveis Instrumentais compostas pelas variáveis exógenas do sistema. Já o método de MCFADDEN é realizado em cinco etapas. Inicialmente, estima-se a função de demanda, em sua forma estrutural original. Em seguida, obtêm-se o valor estimado da quantidade demanda. Posteriormente, estima-se o preço marginal em função da quantidade demanda prevista e do preço efetivo observado. E a última etapa é a estimação da equação de demanda, novamente, com o preço marginal estimado.

Alguns autores, entretanto, apontam que a estimativa por MQO pode ser 
confiável no caso da demanda por água. Por exemplo, pode-se mencionar a semelhança entre as estimativas por MQO e por Variáveis Instrumentais obtidas em Jones \& Morris (1984), Saleth \& Dinar (2000) e Chicoine et al. (1986). Esse último autor estima a função de demanda por água a partir do uso de dados em cross section do consumo mensal no estado de Illinois, sendo que a elasticidade preço da demanda foi estimada em aproximadamente $-0,42 \mathrm{em}$ ambas as metodologias. Denisard et al. (2009) estimaram a elasticidade preço da demanda para os clientes comerciais e industriais da Companhia de Saneamento Básico do Estado de São Paulo (SABESP) também sem considerar modelos alternativos para o viés de simultaneidade. O argumento usado é que o preço é uma variável exógena no problema da firma. Tal consideração leva em conta que a estrutura tarifária é de conhecimento prévio dos agentes no momento da decisão de produção. Assim, a estrutura tarifária é resultado de uma definição institucional dos órgãos de gestão, logo ela pode ser considerada um dado para os consumidores de água. Ou seja, a estrutura tarifária da SABESP tende a ser definida por um processo independente da decisão dos produtores na alocação de seus fatores de produção.

De qualquer modo, para verificar a existência de viés de simultaneidade, este estudo usou o teste de especificação de Hausman. O Apêndice 3 mostra os passos para a aplicação do teste bem como seus resultados, que apontam para a inexistência de viés de simultaneidade no modelo estimado.

\section{Resultados}

A Tabela 2 abaixo mostra os resultados do modelo estimado. Foram utilizadas 3.479 observações para a estimativa dos parâmetros. O resultado do Rquadrado indica que cerca de $70 \%$ da variabilidade da demanda por água pode ser explicada por variações conjuntas dos indicadores utilizados no modelo, o que representa, empiricamente, um poder explicativo alto do modelo especificado. A estatística $\mathrm{F}$ indica a significância conjunta das variáveis. A estatística de Durbin-Watson fica próxima de 2, o que indica a inexistência de autocorrelação dos resíduos.

Porém, o modelo indicou evidências da existência de heterocedasticidade, isto é, a variância dos resíduos não foi constante em relação aos níveis de consumo. Nesse caso, embora não tendenciosos, os estimadores não são os mais eficientes sob o método de mínimos quadrados ordinários (MQO) e, em geral, a inferência pode ficar prejudicada, pois os testes de significância dos parâmetros tendem a ter valores menores (ou iguais) aos estimados sob homocedasticidade (variância constante). Ou seja, os estimadores deixam de ser BLUE (Best Linear Unbiased Estimator), e os resultados para os testes de significância se tornam inexatos (Gujarati 2006). Assim, foi realizado o teste de White para a detecção da heterocedasticidade, e o modelo foi estimado com a correção de White para erros-padrão robustos.

Como resultado, pode-se observar que quase todos os parâmetros estimados foram significativos, a maioria ao nível de $1 \%$ de significância. Em síntese, pode-se inferir que, a cada $1 \%$ de variação do número de domicílios e de crescimento populacional, a demanda por água esteve relacionada a aumentos de, respectivamente, $0,73 \%$ e $0,23 \%$, considerando tudo o mais constante. Embora com o sinal esperado, o consumo de água não foi significativamente influenciado pela temperatura média dos municípios. Isso pode ter ocorrido, 
em grande parte, pela sazonalidade do consumo no Rio Grande do Sul ser maior em função da grande variabilidade climática entre as estações do ano, sendo que, no modelo estimado, em função da limitação temporal de disponibilidade nas diversas bases de informação, os dados utilizados foram anuais, não capturando tal tendência. E, quanto maior o peso da agropecuária e indústria nas estruturas produtivas locais, maior tende a ser a demanda por água. O comportamento do consumo também esteve associado ao nível de escolaridade da população economicamente ativa (PEA), sendo possível inferir que menores consumos estiveram relacionados aos maiores graus de instrução (somente o parâmetro estimado para a variável "ESC3" não foi significativo). A demanda também esteve inversamente relacionada com o nível de renda, pois, em geral, existe uma correlação positiva entre o nível educacional e a renda. Mais especificamente, aumentos de $1 \%$ no peso da agropecuária e da indústria, estiveram associados a elevações de 0,03\% e 0,05\% na demanda por água, respectivamente, ao passo que, para a renda, a expansão de $1 \%$ na mesma esteve relacionada a quedas de $0,10 \%$ no consumo de água.

Como principal conclusão, o parâmetro $\beta_{1}$ de $\mathrm{P}$ estimado foi significativo e, conforme o esperado, inversamente relacionado com o volume consumido de água. Mais precisamente, o modelo estimado indica que, nos municípios gaúchos, a cada $1 \%$ de aumento real da tarifa média de água a demanda tende a cair $0,27 \%$, ao nível de $1 \%$ de significância. Em outros termos, pode-se inferir que, embora de forma inelástica na relação entre consumo e preços, a política tarifária do setor de saneamento tem certa eficácia para ser utilizada como instrumento de gerenciamento da demanda, principalmente em contextos de crise hídrica. Por exemplo, em média, um aumento de $10 \%$ das tarifas cobradas pode gerar reduções no nível de consumo em 2,74\%, considerando tudo o mais constante.

Dada a heterogeneidade das condições ambientais e socioeconômicas dos municípios do estado, e o próprio consumo médio per capita acima das médias estadual e nacional, como já mencionado, foi incluída no modelo a variável dummy "DPOA", com a qual se buscou estimar os efeitos específicos da condução da política tarifária do setor na capital gaúcha. Como resultado, pode-se inferir que, em Porto Alegre, a sensibilidade do consumo em relação a variações reais nas tarifas situa-se em - 0,25 acima daquela estimada para o estado. Mais precisamente, na capital, estima-se que, a cada $1 \%$ de aumento real da tarifa cobrada, a demanda tende a cair $-0,52 \%$, ao nível de $5 \%$ de significância. Portanto, conclui-se que a política tarifária como instrumento de gestão do consumo de água pode ser eficaz também na capital gaúcha.

\section{Considerações Finais}

A gestão da demanda dos recursos hídricos tem papel cada vez mais central, seja em países em desenvolvimento ou desenvolvidos. Em meados de 2014, o centro econômico-financeiro do Brasil viveu uma das maiores crises hídricas da sua história. Esse fato alertou as autoridades brasileiras para a importância do planejamento e da utilização de instrumentos de gestão da demanda em situações de escassez hídrica.

No cenário nacional, o Rio Grande do Sul registra indicadores de consumo levemente inferiores, mas muito próximos aos do país. Mais precisamente em 2016, o Brasil apresentou uma média de consumo per capita de 
Tabela 2: Resultado do modelo estimado (variável dependente $=D_{A}$ )

\begin{tabular}{l|r|r|r|r}
\hline Variáveis & Coeficiente & Erro-padrão & Razão-t & P-valor \\
\hline const & $-1,4537$ & 0,2999 & $-4,8473$ & 0,0000 \\
P & $-0,2747$ & 0,0190 & $-14,4716$ & 0,0000 \\
DOM & 0,7323 & 0,0095 & 76,7401 & 0,0000 \\
POP & 0,2304 & 0,0246 & 9,3665 & 0,0000 \\
T & 0,0068 & 0,0057 & 1,1894 & 0,2348 \\
IND & 0,0468 & 0,0119 & 3,9417 & 0,0001 \\
AGRO & 0,0330 & 0,0163 & 2,0219 & 0,0437 \\
ESC1 & 0,1527 & 0,0266 & 5,7358 & 0,0000 \\
ESC2 & $-0,0736$ & 0,0189 & $-3,8870$ & 0,0001 \\
ESC3 & 0,0174 & 0,0206 & 0,8454 & 0,3983 \\
REM & $-0,0999$ & 0,0284 & $-3,5191$ & 0,0005 \\
DPOA & $-0,2532$ & 0,1139 & $-2,2232$ & 0,0267 \\
\hline Estatística F & & & & 109,2669 \\
P-Valor & & & & 0,0000 \\
R-Quadrado & & & & 0,7030 \\
Durbin-Watson & & & & 2,0246 \\
\hline
\end{tabular}

Fonte: Resultados da pesquisa. Fontes dos dados Brutos: Ver Tabela 1.

água de 153,50 litros/habitante/dia, ao passo que, no estado, tal indicador situou-se em 148,30 litros/habitante/dia. A capital gaúcha, entretanto, teve uma média de consumo preocupante nesse contexto, qual seja, de 220,30 litros/habitante/dia, sendo a segunda capital brasileira com maior consumo per capita (SNIS - Sistema Nacional de Informações sobre Saneamento 2016). Esse nível de demanda chegou a cair nos anos 2001-2009, mas voltou a crescer e, atualmente, apresenta certa estabilidade.

Nesse sentido, seguindo a tendência nacional e, na verdade, mundial, a utilização de instrumentos de gestão do consumo de recursos hídricos no estado e na Capital gaúcha ganhou contornos de importância vital. Entretanto, a análise da eficácia da política tarifária como instrumento de gestão da demanda depende, principalmente, da elasticidade-preço da demanda por água, isto é, de qual a variação do consumo dada uma variação de preços ou, alternativamente, o quão sensível é a quantidade demandada em relação à cobrança. Como se trata de um bem essencial à vida, espera-se que a demanda por água seja relativamente inelástica ao nível de preços. Porém, tal relação pode não ser perfeitamente inelástica (elasticidade-preço igual à zero).

De fato, os resultados dos modelos de dados em painel estimados para todos os municípios gaúchos no período 2010-2016, com dados do SNIS, indicaram que variações reais nas tarifas cobradas podem ter influenciado significativamente os níveis de consumo de água, mesmo este sendo inelástico em relação aos preços. Especificamente, no Rio Grande do Sul, a cada 1\% de aumento real da tarifa média de água, a demanda tende a cair $-0,27 \%$, ao nível de $1 \%$ de significância.

Dada a heterogeneidade ambiental e socioeconômica dos municípios gaúchos, bem como os níveis preocupantes de consumo, buscou-se estimar, incluindo-se no modelo uma variável dummy, essa relação para o caso específico de Porto Alegre. Por conclusão, pode-se observar que, na capital, a sensibilidade do consumo em relação a variações reais nas tarifas situa-se em -0,25 acima daquela estimada para o estado. Mais precisamente, nesse caso, estima-se que, 
a cada $1 \%$ de aumento real da tarifa cobrada, a demanda tende a cair $-0,52 \%$, ao nível de $5 \%$ de significância.

Portanto, pode-se concluir que, tanto no Rio Grande do Sul quanto em Porto Alegre, a determinação dos níveis tarifários pode influenciar significativamente a demanda por água, sendo, em certa medida, um instrumento eficaz para o gerenciamento da utilização dos recursos hídricos.

Por um lado, esses resultados são fundamentais sob o ponto de vista do potencial das políticas públicas. Por outro, é importante lembrar que, sob a ótica do desempenho financeiro das prestadoras, quando o aumento do nível de preços dos serviços tem como objetivo a expansão dos investimentos no setor para universalização dos mesmos, é essencial ponderar que nem todo o aumento das tarifas se converte em elevação da arrecadação, pois os usuários dos serviços tendem a alterar o comportamento do consumo.

\section{Referências Bibliográficas}

Albuquerque, A. P. M. \& Maia, A. A. (2008). O Reconhecimento da Água Bruta como Bem Econômico e a sua Cobrança como Instrumento para uma Efetiva Política Nacional de Recursos Hídricos. In: Anais do XVII Congresso Nacional do CONPEDI, Brasília.

Amaral, A. N. (2000), PhD thesis. Consumo residencial de água tratada em Piracicaba: Uma Aplicação de modelos Temporais. Ano de depósito. 155 p. Tese (Doutorado) - ESALQ/USP.

Asteriou, D. \& G, H. S. (2007). Applied Econometrics. New York: Palgrave Macmillan.

Chicoine, D. L., Deller, S. C. \& Ramamurthy, G. (1986). Water demand estimation under block rate pricing: a simultaneous equation approach. Water Resources Research, v. 22, n. 6, p. 859-863.

Coelli, T. J., Rao, D. S. \& Battese, G. E. (1998). An Introdution to Efficiency and Productivity analysis. 3. ed. London: KluwerAcademicPublishers.

Célia, J. A. V. (2007). Planeta Água Morrendo de Sede: Uma Visão Analítica na Metodologia do Uso e Abuso dos Recursos Hídricos. Porto Alegre: EDPUCRS.

Denisard, C. O. A., Pereda, P. C., Grimaldi, D. \& Fraga, A. (2009). Estimação da Elasticidade-Preço da Demanda dos Clientes Comerciais e Industriais da SABESP. FIPE - Fundação Instituto de Pesquisas Econômicas, São Paulo.

Foster, H. S. \& Beattie, B. R. (1979). Urban residential demand for water in the United States. Land Economics, v. 55, n. 1, p. 43-58.

Gujarati, D. N. (2006). Econometria Básica. Rio de Janeiro: Campus/Elsevier.

Hill, R. C., Griffiths, W. E. \& Judge, G. G. (1999). Econometria. São Paulo: Saraiva.

Jones, C. V. \& Morris, J. R. (1984). Instrumental price estimates and residential water demand. Water Resources Research, v. 20, n. 2, p. 197-202. 
Pizaia, M. G. \& Alves, R. (2008). O esquema de tarifas em bloco praticado pelas companhias de água: um estudo dos consumidores residenciais de baixa renda. In: XLVI Congresso da Sociedade Brasileira de Economia, Administração e Sociologia Rural SOBER, Rio Branco.

Rosa, A. L. T., Fontenele, R. E. \& Nogueira, C. A. G. (2006). Estimativa da Demanda de Água Residencial Urbana no Estado do Ceará. In: $30^{\circ}$ Encontro Nacional da ANPAD, Salvador, set.

Saleth, R. M. \& Dinar, A. (2000). Urban thirst: water supply augmentation and pricing policy in Hyderabad city. The World Bank Technical Paper, India, n. 395.

Schneider, M. L. \& Whitlach, E. E. (1991). User-specific water demand elasticities. Journal of Water Resources Planning and Management, v. 117, n. 1, p. 52-73.

SNIS - Sistema Nacional de Informações sobre Saneamento (2016). Diagnóstico dos Serviços de Água e Esgotos, 2010-2016. Disponível em: http: www.snis.gov.br. Acesso em: 18 jan. 2019.

Varian, H. R. (1990). Microeconomic analysis. 2. ed. New York: W.W. Norton \& Company, $559 \mathrm{p}$.

Wong, S. T. (1972). A model on municipal water demand: a case study of Northeastern Illinois. Land Economics, v. 48, n. 1, p. 34-44.

Wooldridge, J. M. (2002). Econometric analysis of cross section and panel data. Cambridge, Massachusetts: MIT Press, 206 p.

Worthington, A. C. \& Hoffman, M. (2008). An empirical survey of residential water demand modeling. Journal of Economic Surveys, v. 22, n. 5, p. 842-871.

Young, R. A. (1973). Price elasticity of demand for municipal water: a case study of Tucson, Arizona. Water Resources Research, v. 9, n. 4, p. 1068-1072.

\section{Apêndice $A$}


Figura A.1: Mapa quantílico dos municípios gaúchos, classificados em 5 faixas de consumo médio per capita de água - 2016

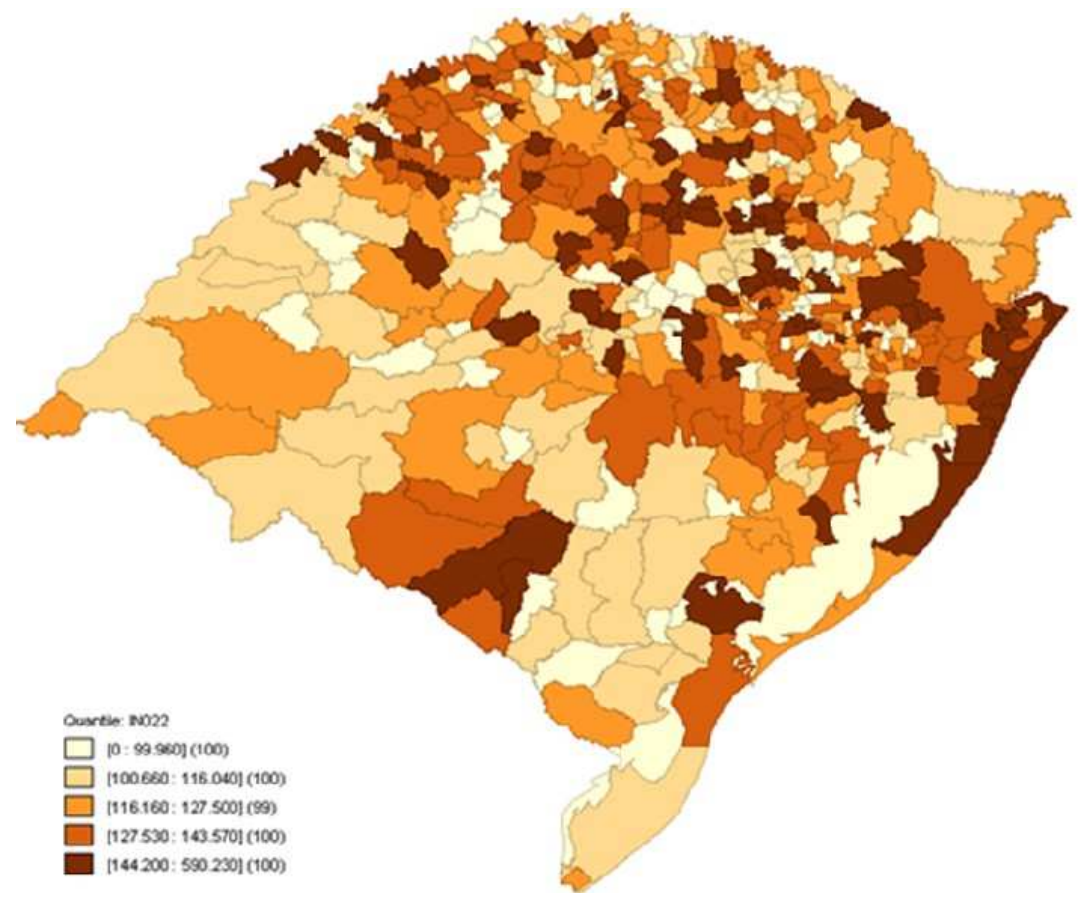

Fonte: Sistema Nacional de Informações sobre Saneamento (SNIS)

\section{Apêndice B}


Figura B.1: Mapa quantílico dos municípios gaúchos, classificados em 5 faixas de consumo em relação à disponibilidade de água, por economia 2016

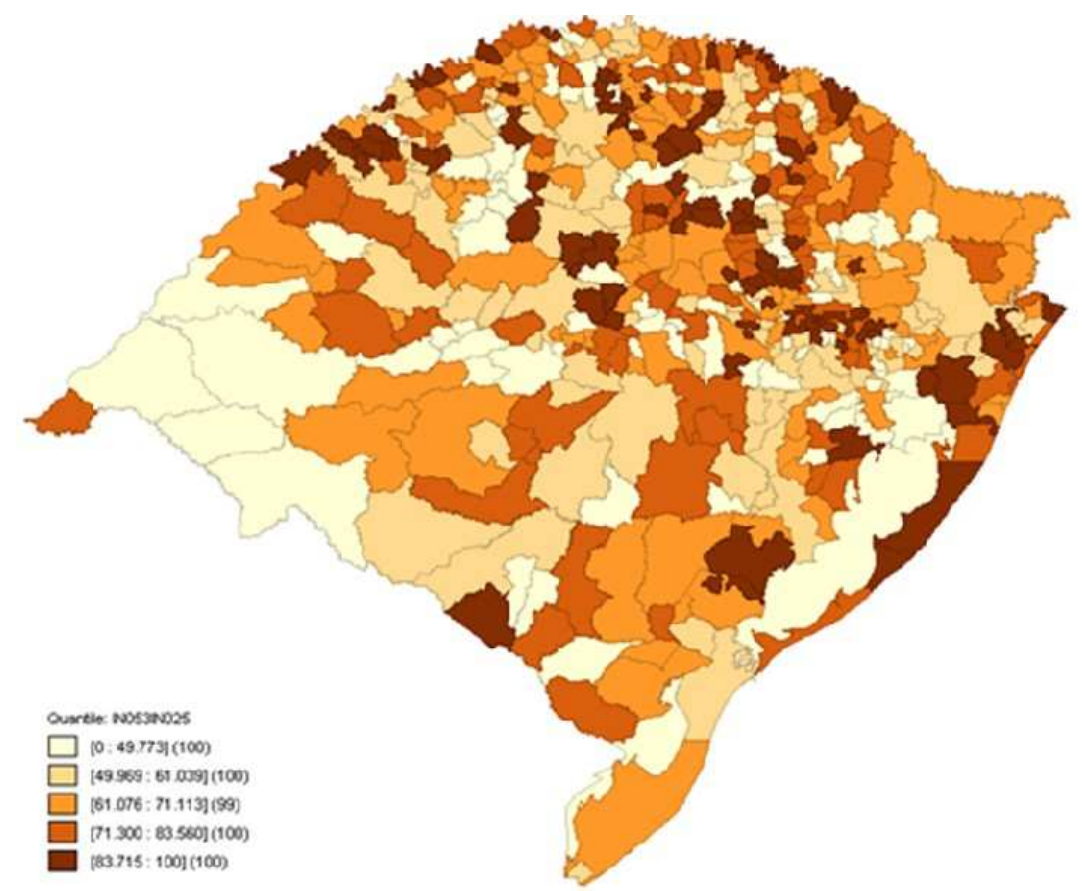

Fonte: Sistema Nacional de Informações sobre Saneamento (SNIS)

\section{Apêndice C : Teste de Hausman para a simultaneidade}

No modelo especificado, supõe-se que as variáveis " $D_{A}$ " e " $P$ " são endógenas, dado que tanto o preço influencia a demanda, quanto a quantidade ofertada tende a se relacionar com o preço, sendo as demais variáveis exógenas. Assim, o teste de erro de especificação de Hausmann consiste em, primeiramente, estimar o modelo com duas equações, uma de oferta e outra de demanda por água. As variáveis usadas no teste estão definidas na Tabela 1.

Função de demanda:

$$
\begin{aligned}
\ln \left(D_{A i t}\right)=C_{i}+ & \beta_{1} \ln \left(P_{i t}\right)+\beta_{2} \ln \left(D O M_{i t}\right)+\beta_{3} \ln \left(P O P_{i t}\right)+\beta_{4} \ln \left(T_{i t}\right) \\
+ & \beta_{5} \ln \left(I N D_{i t}\right)+\beta_{6} \ln \left(A G R O_{i t}\right)+\beta_{7} \ln \left(E S C 1_{i t}\right) \\
+ & \beta_{8} \ln \left(E S C 2_{i t}\right)+\beta_{9} \ln \left(E S C 3_{i t}\right)+\beta_{10} \ln \left(R E M_{i t}\right) \\
& +\beta_{11} \ln \left(D P O A * P_{i t}\right)+u_{1 i t}
\end{aligned}
$$

Função de oferta:

$$
\ln \left(Q A_{i t}\right)=c_{i}+\alpha_{1} \ln \left(P_{i t}\right)+u_{2 i t}
$$


Se não houver problema de simultaneidade, isto é, caso em que preços e quantidades não são mutuamente dependentes, " $P_{i t}$ " e " $u_{2 i t}$ " não devem ser correlacionados. De outra parte, se houver simultaneidade, " $P_{i t}$ " e " $u_{2 i t}$ " são correlacionados. Para verificar tal correlação, o teste de Hausman segue as etapas a seguir.

Primeiro, estima-se as seguintes equações na forma reduzida, ou seja, as equações com as variáveis endógenas em função das exógenas.

$$
\begin{aligned}
\ln \left(P_{A i t}\right)= & a_{i}+\infty_{1} \ln \left(D O M_{i t}\right)+\infty_{2} \ln \left(P O P_{i t}\right)+\infty_{3} \ln \left(T_{i t}\right) \\
+ & \infty_{4} \ln \left(I N D_{i t}\right)+\infty_{5} \ln \left(A G R O_{i t}\right)+\infty_{6} \ln \left(E S C 1_{i t}\right) \\
+\infty_{7} & \ln \left(E S C 2_{i t}\right)+\infty_{8} \ln \left(E S C 3_{i t}\right)+\infty_{9} \ln \left(R E M_{i t}\right) \\
& +\infty_{10} \ln \left(D P O A * P_{i t}\right)+v_{1 i t} \\
\ln \left(D_{A i t}\right)= & b_{i}+\gamma_{1} \ln \left(D O M_{i t}\right)+\gamma_{2} \ln \left(P O P_{i t}\right)+\gamma_{3} \ln \left(T_{i t}\right) \\
+ & \gamma_{4} \ln \left(I N D_{i t}\right)+\gamma_{5} \ln \left(A G R O_{i t}\right)+\gamma_{6} \ln \left(E S C 1_{i t}\right) \\
& +\gamma_{7} \ln \left(E S C 2_{i t}\right)+\gamma_{8} \ln \left(E S C 3_{i t}\right)+\gamma_{9} \ln \left(R E M_{i t}\right) \\
& +\gamma_{10} \ln \left(D P O A * P_{i t}\right)+v_{2 i t}
\end{aligned}
$$

em que $v_{1 i t}$ e $v_{2 i t}$ são os termos de erro na forma reduzida.

Assim, obtêm-se por MQO a seguinte equação na forma reduzida:

$$
\begin{aligned}
\hat{P}_{i t}=\hat{a}_{i}+ & \hat{\infty}_{1} \ln \left(D O M_{i t}\right)+\hat{\infty}_{2} \ln \left(P O P_{i t}\right)+\hat{\infty}_{3} \ln \left(T_{i t}\right) \\
+ & \hat{\infty}_{4} \ln \left(I N D_{i t}\right)+\hat{\infty}_{5} \ln \left(A G R O_{i t}\right)+\hat{\infty}_{6} \ln \left(E S C 1_{i t}\right) \\
+ & \hat{\infty}_{7} \ln \left(E S C 2_{i t}\right)+\hat{\infty}_{8} \ln \left(E S C 3_{i t}\right)+\hat{\infty}_{9} \ln \left(R E M_{i t}\right) \\
& +\hat{\infty}_{10} \ln \left(D P O A * P_{i t}\right)+\hat{v}_{1 i t}
\end{aligned}
$$

Onde:

$$
P_{i t}=\hat{P}_{i t}+\hat{v}_{1 i t}
$$

Substituindo $P_{i t}$ na função de oferta tem-se que:

$$
\ln \left(Q_{A i t}\right)=c_{i}+\phi_{1} \hat{P}_{i t}+\phi_{2} \hat{v}_{1 i t}+u_{2 i t}
$$

em que a significância estatística de $\phi_{2}$ determina a existência de simultaneidade.

Os resultados para os coeficientes estimados são mostrados a seguir. 
Tabela C.1: Resultados do teste de Hausman

\begin{tabular}{|c|c|c|c|c|}
\hline & Coeficiente & Erro-padrão & Razão-t & P-valor \\
\hline const & $-1,5231$ & 0,0122 & $-124,3921$ & 0,0000 \\
\hline$\hat{P}$ & 9,8816 & 0,0172 & 575,4087 & 0,0000 \\
\hline$\hat{V}_{1}$ & 0,0012 & 0,0072 & 0,1612 & 0,2389 \\
\hline
\end{tabular}

Conclusão: Não se pode rejeitar a hipótese nula de que $\phi_{2}=0$, ou seja, os resultados apontam para a inexistência de simultaneidade. 
